\title{
MICOFENOLATO DE MOFETIL VS MICOFENOLATO DE SÓDIO APÓS TRANSPLANTE SIMULTÂNEO DE PÂNCREAS-RIM (TSPR)
}

\author{
MYCOPHENOLATO MOFETIL VS MYCOPHENOLATE SODIUM AFTER SIMULTANEOUS PANCREAS- \\ KIDNEY TRANSPLANTATION (SPKT)
}

\author{
Erika Bevilaqua Rangel', Cláudio Santiago Melaragno', João Roberto de Sá2, Adriano Miziara Gonzalez³, \\ Marcelo Moura Linhares ${ }^{3}$, Alcides Salzedas ${ }^{3}$, José Osmar Medina-Pestana'
}

\begin{abstract}
RESUMO
Objetivos: Os sintomas gastrointestinais são freqüentes após o uso de derivados do ácido micofenólico. Os objetivos do trabalho foram relatar a incidência de diarréia aguda e descrever os fatores de risco associados a sua ocorrência após o transplante simultâneo de pâncreasrim (TSPR). Métodos: Inclusão de 165 pacientes submetidos a TSPR no período de Dez/00 a Maio/07. Realizada regressão logística binária, de modo que a ocorrência de diarréia aguda foi a variável dependente; as variáveis independentes incluíram idade, sexo, raça, tempo de diabetes e diálise, modalidade de diálise, ocorrência de função retardada do enxerto renal e uso de Micofenolato de Mofetil (MMF) vs Micofenolato de Sódio (MPS). P < 0,05 foi considerado significante. Resultados: As médias de idade, tempo de diálise e tempo de diabetes foram: $34,9 \pm 8,2$ anos, $27,3 \pm 18,3$ meses e 21,9 $\pm 16,2$ anos. $63 \%$ dos pacientes usaram MMF, 36,4\% usaram MPS e $0,6 \%$ usaram Azatioprina. A análise multivariada mostrou que o tempo de diabetes ( $\mathrm{p}=0,049, \mathrm{IC} 95 \% 1,0-1,13)$ e uso de MMF ( $\mathrm{p}=0,013$, IC 95\% 0,2-0,82) foram os principais determinantes da diarréia aguda não-infecciosa após o TSPR. O MMF esteve associado à maior necessidade de redução da dose $(79,2 \%$ vs $62,3 \%, \mathrm{p}=0,024)$ e a episódios mais severos de diarréia aguda associados à hipotensão ortostática (42,4\% vs $15,1 \%, p=0,001)$ do que o MPS, respectivamente. Não houve diferença entre o MMF e o MPS em relação à ocorrência de infecção por citomegalovírus $(36,5 \%$ vs $32,8 \%, p=0,6)$ e de rejeição aguda do enxerto renal após redução da dose $(30,8 \%$ vs $26,7 \%, p=0,53)$, respectivamente. Conclusões: A diarréia aguda após o TSPR está relacionada ao tempo de diabetes e ao uso de MMF. O uso preferencial de MPS está associado à menor necessidade de redução da dose e a episódios menos severos de diarréia aguda. No entanto, a ocorrência de rejeição aguda do enxerto renal após redução da dose é similar entre o MMF e o MPS.
\end{abstract}

Descritores: Transplante de Pâncreas; Imunossupressão; Diarréia

\footnotetext{
Instituições:

1 Departamento de Nefrologia, Universidade Federal de São Paulo - Brasil

2 Departamento de Endocrinologia, Universidade Federal de São Paulo - Brasil

${ }^{3}$ Departamento de Cirurgia, Universidade Federal de São Paulo - Brasil
}

\section{Correspondência:}

Érika B Rangel, Departamento de Nefrologia - Universidade Federal de São Paulo

Rua Botucatu, 740 - São Paulo / SP - CEP: 04023-900 - Brasil

Tel.: (11) 55746300 / Fax: (11) 55739652

E-mail: erikabr@uol.com.br

Recebido em: 02.01.2009

Aceito em: 16.01.2009

\section{INTRODUÇÃO}

O transplante simultâneo de pâncreas-rim (TSPR) é um tratamento efetivo para pacientes com diabetes mellitus insulino-dependente associado à insuficiência renal crônica.

A imunossupressão de escolha após o TSPR inclui a utilização de Tacrolimus e Micofenolato, além da indução com anticorpos depletores ou não-depletores de linfócitos. ${ }^{1}$

A introdução de Micofenolato de Mofetil (MMF) ao esquema imunossupressor correlaciona-se tanto à redução da incidência de rejeição aguda no primeiro ano ${ }^{2}$ quanto da nefropatia crônica do enxerto em $27 \% 3$ após transplante renal.

O Micofenolato inibe a enzima inosina 5'-monofosfato desidrogenase, que é uma enzima chave para a via de novo da síntese das purinas, causando supressão seletiva dos linfócitos.

Apesar de altamente efetivo, o MMF está associado a adversos eventos gastrointestinais frequentes como diarréia aguda, além de leucopenia. ${ }^{4}$ Quando há redução ou descontinuação da dose do Micofenolato devido à ocorrência de sintomas gastrointestinais, 
estima-se que o risco de rejeição aguda aumente em 2,36 e 2,72 vezes, respectivamente. ${ }^{5}$

Os objetivos do estudo foram relatar a incidência de diarréia aguda, descrever os fatores de risco associados a sua ocorrência e comparar a severidade da diarréia aguda entre o Micofenolato de Mofetil (MMF) e o Micofenolato de Sódio (MPS) após o TSPR.

\section{PACIENTES E MÉTODOS}

No período de dezembro de 2000 a maio de 2007 foram realizados 166 transplantes simultâneos de pâncreas-rim (TSPR) na UNIFESP-EPM.

Os critérios de elegibilidade para realização do TSPR foram: idade menor do que 55 anos, baixo risco cardiovascular, ausência de amputações secundárias à doença vascular periférica associada ao DM, aderência ao uso de medicações e orientações médicas, compreensão do procedimento, presença de duas ou mais complicações relacionadas ao DM (nefropatia, retinopatia e/ou neuropatia) e índice de massa corpórea abaixo de $32 \mathrm{~kg} / \mathrm{m}^{2}$. Além disso, pacientes com infecções ativas, neoplasias e história de etilismo atual foram excluídos temporariamente ou definitivamente da lista de espera. Foram incluídos pacientes em diálise ou em pré-dialise com clearance de creatinina menor ou igual a $20 \mathrm{ml} / \mathrm{min} / 1,73 \mathrm{~m}^{2}$.

As técnicas cirúrgicas incluíram derivação exócrina pancreática entérica em 158 casos e vesical em sete casos. A reconstrução arterial foi realizada com enxerto em "Y" da artéria ilíaca do doador através da conecção da artéria ilíaca externa à artéria mesentérica superior e da artéria ilíaca interna à artéria esplênica, respectivamente. A anastomose vascular venosa foi realizada entre a veia porta do doador e a veia ilíaca comum ou cava do receptor. A imunossupressão inicial incluiu Tacrolimus $0,15 \mathrm{mg} / \mathrm{kg} /$ dose, ajustado de acordo com o tempo pós-transplante (nível sérico de 10-15 ng/ml nos primeiros 30 dias, 8-10 ng/ml entre 31 e 90 dias e 5-10 ng/ml posteriormente), Prednisona 0,5 mg/kg/dia (máximo de $30 \mathrm{mg} /$ dia e redução de 5-10 mg a cada mês até a dose de manutenção de $5 \mathrm{mg} /$ dia a partir do sexto ao oitavo mês do transplante) e Micofenolato de Mofetil 2 g/dia ou Micofenolato de Sódio 1,44 g/dia em todos os casos.

Houve duas mudanças no esquema de imunossupressão após o TSPR na UNIFESP-EPM. A primeira mudança ocorreu em novembro de 2001, quando a Ciclosporina foi substituída pelo Tacrolimus, de modo que 16 pacientes utilizaram Ciclosporina associada a Prednisona e ao Micofenolato de Mofetil. A segunda mudança ocorreu a partir de janeiro de 2005, quando o Micofenolato de Mofetil foi substituído pelo Micofenolato de Sódio, de modo que 105 pacientes utilizaram Tacrolimus ou Ciclosporina, Prednisona e Micofenolato de Mofetil e 60 pacientes utilizaram Tacrolimus, Prednisona e Micofenolato de Sódio. Assim sendo, o Micofenolato de Mofetil foi utilizado em $63 \%$ dos pacientes, o Micofenolato de Sódio em 36,4\% e a Azatioprina em 0,6\%. O único paciente que estava em uso de Azatioprina foi excluído do trabalho.

A indução com anticorpo mono- ou policlonal não foi rotineiramente utilizada, mas apenas para casos de re-transplante ou com painel acima de $20 \%$ ou em alguns casos em que o tempo de isquemia fria do rim excedeu 24 horas ou houve oligúria no pós-operatório imediato. A função retardada do enxerto renal foi definida como necessidade de diálise na primeira semana após o transplante.

Foi realizada regressão logística binária, de modo que a ocorrência de diarréia aguda (definida como aumento do número de evacuações de causa não-infecciosa, sendo excluídas as causas infecciosas bacterianas, parasitárias, virais ou fúngicas) foi a variável dependente e as variáveis independentes incluíram idade, sexo, raça, tempo de diabetes e diálise, modalidade de diálise, ocorrência de função retardada do enxerto renal e uso de MMF vs MMS. A intensidade da diarréia foi definida como desidratação associada ou não à hipotensão arterial ortostática (definida como a queda da pressão arterial sistólica maior do que $20 \mathrm{mmHg}$ ou da pressão diastólica maior do que $10 \mathrm{mmHg}$ em resposta à mudança postural na posição deitada para a ortostática depois de um minuto) e com necessidade de hidratação endovenosa com solução salina a $0,9 \%$. A infecção pelo citomegalovírus foi diagnosticada pelo exame de antigenemia (detecção da proteína pp65 em leucócitos do sangue periférico). O diagnóstico de rejeição aguda do enxerto renal foi confirmado pela biópsia percutânea (critérios de Banff 2005), sendo o critério para a sua realização o aumento da creatinina.

Foi utilizado o intervalo de confiança de $95 \%$ e determinada a razão de chances. Considerado significante o $P<0,05$.

\section{RESULTADOS}

As médias de idade, tempo de diálise e de tempo de diabetes foram 34,9 \pm 8,2 anos, 27,3 \pm 18,3 meses e 21,9 $\pm 16,2$ anos, respectivamente.

Em relação às diferenças entre uso de MMF e de MPS, destaca-se maior incidência de diarréia aguda com o MMF em comparação ao MPS (79,2\% vs 62,3\%, respectivamente, $P=0,024)$, havendo necessidade de redução da dose de MMF ou de MMS em torno de $50-75 \%$. A redução da dose de MMF ou de MPS ocorreu inicialmente 23,7 \pm 48,7 dias (mediana 7 dias) após o TSPR. Alguns pacientes necessitaram de uma segunda redução da dose daquelas medicações, que ocorreu 72,4 \pm 119,6 dias (mediana 20 dias) após o TSPR.

Além disto, houve maior incidência de desidratação associada à hipotensão arterial ortostática, requerendo hidratação endovenosa com solução salina a $0,9 \%$ com MMF, em comparação ao MPS (42,4\% vs $15,1 \%$, respectivamente, $P=0,001)$. Em nenhum paciente a dose de MMF ou de MMS foi aumentada após ter sido reduzida devido à diarréia aguda ou hipotensão arterial.

A análise univariada mostrou que o tempo de diabetes $(P=0,046$, IC 95\% 1,0-1,13) e o uso de MMF vs MPS ( $P=0,027$, IC 95\% $0,24-0,92$ ) (Tabela 1 ) foram os principais fatores de risco para a ocorrência de diarréia aguda não-infecciosa após o TSPR. O sexo, idade, raça, tempo de diálise e modalidade de diálise, além da ocorrência de função retardada do enxerto renal não tiveram impacto no diagnóstico de diarréia aguda.

Da mesma forma, a análise multivariada mostrou que o tempo de diabetes, apesar da correlação marginal ( $P=0,049$, IC 95\% 1,0-1,13), e do uso de $\operatorname{MMF}(P=0,013$, IC $95 \%$ 0,2-0,82) configuram-se como fatores de risco para a ocorrência de diarréia aguda não-infecciosa após o TSPR (Tabela 2). No entanto, não houve diferença entre o tempo médio de história de diabetes entre os grupos MMF e MPS $(21,8 \pm 5,9$ anos vs 22,05 $\pm 6,6$ anos, respectivamente, $P=0,79$, IC $95 \%-2,4-1,8)$.

Não houve diferença também em relação à ocorrência de infecção por citomegalovírus quando utilizade MMF e MPS (36,5\% vs $32,8 \%$, respectivamente, $P=0,6$ ) e nem na ocorrência de rejeição aguda do enxerto renal após redução da dose de MMF e de MPS 
Tabela 1. Análise univariada dos fatores de risco para ocorrência de diarréia aguda não-infecciosa após o transplante simultâneo de pâncreas-rim.

\begin{tabular}{lcccc}
\hline & $\boldsymbol{P}$ & $\begin{array}{c}\text { razão de } \\
\text { chances }\end{array}$ & \multicolumn{2}{c}{ IC } \\
\hline Sexo & & & inferior & superior \\
Raça & 0,19 & 0,64 & 0,33 & 1,24 \\
Idade & 0,19 & 1,7 & 0,77 & 3,78 \\
Micofenolato de Mofetil vs & 0,37 & 1,02 & 0,98 & 1,06 \\
Micofenolato de Sódio & 0,027 & 0,47 & 0,24 & 0,92 \\
Tempo de Diabetes & & & & 1,06 \\
Tempo de Diálise & 0,046 & 1,06 & 1,0 & 1,13 \\
Hemodiálise vs Diálise Peritoneal & 0,37 & 1,0 & 0,99 & 1,03 \\
Função retardada do enxerto renal & 0,44 & 1,43 & 0,58 & 3,56 \\
\hline
\end{tabular}

Tabela 2. Análise multivariada dos fatores de risco para ocorrência de diarréia aguda não-infecciosa após o transplante simultâneo de pâncreas-rim.

\begin{tabular}{lcccc}
\hline & P & $\begin{array}{c}\text { razão de } \\
\text { chances }\end{array}$ & \multicolumn{2}{c}{ IC } \\
\hline & & & inferior & superior \\
\hline $\begin{array}{l}\text { Micofenolato de Mofetil vs Micofeno- } \\
\text { lato de Sódio }\end{array}$ & 0,013 & 0,39 & 0,2 & 0,82 \\
Tempo de Diabetes & 0,049 & 1,06 & 1,0 & 1,13 \\
\hline
\end{tabular}

(30,8\% vs $26,7 \%$, respectivamente, $\mathrm{P}=0,53)$. A rejeição aguda do enxerto renal ocorreu após 8,6 4,5 dias (mediana de 8 dias) após redução da dose de MMF ou de MPS.

Seis pacientes realizaram conversão de MMF para o MPS após 100,4 \pm 136,6 dias (mediana 60 dias) devido à diarréia aguda persistente mesmo após redução de 50\% da dose de MMF. Em um daqueles seis pacientes houve necessidade de suspensão do MPS devido à persistência de diarréia aguda, mesmo após exaustiva investigação (exame parasitológico de fezes, coprocultura, investigação de citomegalovírus e colonoscopia), sendo introduzida a Azatioprina.

\section{DISCUSSÃO}

No presente trabalho, a ocorrência de diarréia aguda de etiologia não infecciosa após transplante simultâneo de pâncreas-rim (TSPR) esteve relacionada ao uso de Micofenolato de Mofetil (MMF) em comparação ao Micofenolato de Sódio (MPS) e ao tempo de história de diabetes.

Na literatura, a substituição da Azatioprina pelo MMF mostrou que esse último está associado à menor taxa cumulativa de rejeição aguda nos três primeiros anos após transplante renal. ${ }^{2}$ Além disso, os dados do USRDS (United States Renal Data System) mostram que o MMF está relacionado à maior sobrevida do paciente e do enxerto renal, especialmente em pacientes negros americanos. ${ }^{6,7}$ Após o transplante de pâncreas, o MMF está também associado à menor taxa de rejeição aguda no primeiro ano, em comparação à Azatioprina ( $15 \%$ vs $43 \%$, respectivamente), incluindo o TSPR, transplante de pâncreas após rim e transplante de pâncreas solitário, embora a sobrevida do transplante de pâncreas no primeiro ano não tenha sido diferente. ${ }^{1}$

Na literatura, a incidência de diarréia aguda com o esquema MMF e Tacrolimus varia de 29 a $64 \%{ }^{8,9}$ Cerca de $60 \%$ dos pacientes em uso de MMF necessitaram de redução da dose devido à ocorrência de eventos adversos, sendo as causas mais freqüentes a leucopenia e diarréia aguda ( $55 \%$ e $22 \%$, respectivamente).$^{10}$

Conforme revisado por Davies e cols, várias são as causas de diarréia aguda após o transplante, incluindo-se causas infecciosas, como bacterianas, virais, fúngicas e secundária a parasitas e a causas não infecciosas, como a imunossupressão e comorbidades prévias ao transplante, como diabetes, intolerância à lactose, dentre outras ${ }^{11}$. Os fatores de risco para diarréia aguda descritos por outros autores incluem: sexo feminino, presença de diabetes tipo1 e esquema imunossupressor envolvendo MMF e Tacrolimus. ${ }^{12}$ Desta forma, a elevada incidência de diarréia aguda no presente trabalho pode ser explicada pela escolha da imunossupressão, isto é, MMF ou MPS associado a Tacrolimus e pelo fato de todos os pacientes terem história pregressa de diabetes.

A neuropatia autonômica gastrointestinal pode se manifestar por diarréia em até $20 \%$ dos pacientes diabéticos, sendo secundária à disfunção da motilidade do intestino delgado, que favorece o crescimento bacteriano devido à estase, ou ao aumento da motilidade e atividade secretória. ${ }^{13}$ Geralmente, a diarréia é intermitente, mas pode se manifestar com mais episódios de 20 evacuações diárias, geralmente de aspecto líquido, o que torna o diagnóstico diferencial com a diarréia aguda secundária ao uso do Micofenolato extremamente difícil. Desta forma, sugere-se que o tempo de história de diabetes observado em nossos pacientes (21,9 anos) pode ter contribuído para a ocorrência mais freqüente de diarréia tanto no grupo MMF quanto MPS, uma vez que esse tempo foi similar nos dois grupos. Além disso, o diagnóstico de desidratação esteve associado ao quadro de hipotensão ortostática na maior parte dos nossos casos (42,4\% com MMF e 15,1\% com MPS). Nos pacientes diabéticos, a hipotensão ortostática é geralmente decorrente de lesão nas fibras vasomotoras simpáticas eferentes, especialmente na vasculatura esplânica, além da redução das resistências vasculares cutânea, esplânica e total. ${ }^{13}$ Além disso, a hipotensão ortostática pode refletir o efeito hipotensor da insulina, que pode ter sido por sua vez exacerbado pelo fato dos pacientes serem hiperinsulinêmicos devido à drenagem endócrina sistêmica após o TSPR. A hipotensão ortostática pode também ser explicada pela variação dos valores da glicemia pós-prandial e pelas variações nos padrões de retenção de líquidos devido à insuficiência renal ou insuficiência cardíaca congestiva. ${ }^{13}$ Após o TSPR, é relatada incidência de $28 \%$ de hipotensão ortostática. ${ }^{14}$ Seu tratamento pode ser realizado com Fludrocortisona ${ }^{13}$ ou com Midodrina. ${ }^{14}$

Apesar de não haver trabalhos randomizados na literatura sobre a maior eficiência de MPS em comparação ao MMF, sugere-se que após transplante renal a redução da dose de MPS seja menos freqüente em comparação ao MMF. ${ }^{15}$ Além disso, a severidade dos eventos adversos gastrointestinais atribuídos ao MMF é reduzida após a conversão para MPS, ${ }^{16}$ o que estaria de acordo com nossos dados.

O MPS apresenta um revestimento que se dissolve no $\mathrm{pH}$ acima de 5, o que permite a liberação do ácido micofenólico no intestino delgado com perfil farmacocinético mais tardio que o MMF (2-2,5 horas vs $0,75-1$ hora, respectivamente). ${ }^{17,18}$ No entanto, tanto o 
MPS quanto o MMF apresentam a mesma exposição aos seus derivados, como o ácido micofenólico e o glucoronide do ácido micofenólico. ${ }^{17,18}$ Provavelmente, devido àquela diferença no perfil farmacocinético, alguns autores sugerem que a conversão de MMF para MPS após transplante renal possa ser uma alternativa para pacientes que apresentem sintomas gastrointestinais, uma vez que ocorre melhora desses sintomas ${ }^{19}$, melhorando a qualidade de vida. ${ }^{19,20}$ Cerca de $10 \%(n=6)$ de nossos pacientes em uso de MMF foram convertidos para MPS, na tentativa de controlar os episódios de diarréia aguda, não havendo nenhum caso de rejeição aguda após tal conversão. Conforme descrito por outros autores, a conversão de MMF para MPS em pacientes usando também Tacrolimus é um procedimento seguro, uma vez que tanto a exposição ao ácido micofenólico quanto os efeitos farmacodinâmicos são similares, não sendo comprometida a eficácia terapêutica. ${ }^{21}$ Apenas um paciente persistiu com diarréia após a conversão para MPS, e optou-se pela conversão para Azatioprina. Na literatura, é descrita a conversão de MMF para Azatioprina no primeiro ano em 14\% dos pacientes após o TSPR, em $26 \%$ dos pacientes submetidos ao transplante de pâncreas após rim e em 39\% dos pacientes submetidos ao transplante de pâncreas isolado. ${ }^{1}$ Além disso, a redução ou a interrupção da administração de MMF após o transplante renal devido à presença dos sintomas gastrointestinais estão associados tanto à rejeição aguda quanto à perda do enxerto renal. ${ }^{5,21}$ Cerca de 1/3 de nossos pacientes apresentaram rejeição aguda renal após redução do MMF ou do MPS, embora nenhum deles tenha perdido o enxerto renal. Contudo, o impacto na sobrevida dos enxertos ainda está sendo estudado em nosso centro.

Apesar de não termos avaliado o impacto econômico, outros autores relataram aumento do custo, tanto com a redução quanto com a descontinuação do $\mathrm{MMF}^{22}$

Além disso, não encontramos diferença em relação à ocorrência de infecção por citomegalovírus entre o MMF e o MPS, de modo que a incidência encontrada está de acordo com a literatura. ${ }^{23}$

O uso preferencial de MPS pode ser estratégico após o TSPR, uma vez que seu uso está associado à menor necessidade de redução da dose devido à ocorrência de diarréia aguda não-infecciosa, bem como a episódios menos severos desse evento adverso. Estas observações nos motivaram a utilizar o MPS em detrimento do MMF após o TSPR na UNIFES-EPM, embora estudos randomizados e controlados sejam necessários para confirmar nossos achados. Além disso, o diagnóstico de rejeição aguda do enxerto renal após redução tanto de MMF quanto de MPS é similar e deve ser investigado.

\section{CONCLUSÕES}

A diarréia aguda após o TSPR está relacionada ao tempo de diabetes e ao uso de MMF. O uso preferencial de MPS está associado à menor necessidade de redução da dose e a episódios menos severos de diarréia aguda. No entanto, a ocorrência de rejeição aguda do enxerto renal após redução da dose é similar entre o MMF e MPS.

\section{ABSTRACT}

Purpose: Adverse gastrointestinal events are frequent after the use of Mycophenolate. The purposes of the present study were to report the incidence of acute non-infectious diarrhea, to determine the risk factors, and make a comparison on the severity between Mycophenolate Mofetil (MMF) and Enteric-Coated Mycophenolate Sodium (EC-MPS) after simultaneous pancreas-kidney transplantation (SPKT). Methods: It was included 165 SPKT patients from Dec/00 to May/07. Uni-and multivariate analyses were performed, being acute noninfectious diarrhea the dependent variable. $\mathrm{P}<0.05$ was significant. Results: Mean age, duration of dialysis and diabetes were $34.9 \pm$ 8.2 years, $27.3 \pm 18.3$ months and $21.9 \pm 16.2$ years, respectively. $63 \%$ used MMF, 36.4\% used EC-MPS, and $0.6 \%$ used Azathioprine. Multivariate analysis showed that the duration of diabetes $(P=0.049, \mathrm{CI} 95 \% 1.0-1.13)$ and the use of MMF $(P=0.013, \mathrm{CI} 95 \% 0.2-0.82)$ were the major determinants to post-SPKT acute diarrhea. Dose reduction of MMF $(79.2 \%$ vs $62.3 \%, \mathrm{P}=0.024)$ and the severity of diarrhea associated to orthostatic hypotension were more pronounced with MMF than with EC-MPS (42.4\% vs $15.1 \%$, P = 0.001). There was no difference between MMF and EC-MPS after the dose reduction related to the occurrence of acute kidney rejection $(30.8 \%$ vs $26.7 \%$, $\mathrm{P}=$ 0.53). Conclusions: Post-SPKT acute non-infectious diarrhea is related to the duration of diabetes and to MMF. The preferential use of EC-MPS is associated to a lower need of dose reduction and less severe episodes of acute diarrhea compared to MMF, although the dose reduction was equally associated to acute kidney rejection.

Keywords: Pancreas Transplantation; Immunosuppression; Diarrhea

\section{REFERÊNCIAS}

1. Gruessner RW, Sutherland DE, Drangstveit MB, Wrenshall L, Humar A, Gruessner AC. Mycophenolate mofetil in pancreas transplantation. Transplantation. 1998;66(3):318-23.

2. Mycophenolate mofetil for treatment of a first acute renal allograft rejection: a threeyear follow-up. The Mycophenolate Mofetil Acute Renal Rejection Study Group. Transplantation. 2001;71(8):1091-7.

3. Ojo AO, Meier-Kriesche HU, Hanson JA, Leichtman AB, Cibrik D, Magee JC, et al. Mycophenolate Mofetil reduces late renal allograft loss independent of acute rejection. Transplantation. 2000;69(11):2239-40.
4. Behrend M. Adverse gastrintestinal effects of mycophenolate Mofetil: aetiology, incidence, and management. Drug Saf. 2001;24(9):645-63.

5. Bunnapradist S, Lentine KL, Burroughs TE, Pinsky BW, Hardinger KL, Brennan DC, et al. Mycophenolate Mofetil dose reductions and discontinuations after gastrointestinal complications are associated with renal transplant graft failure. Transplantation. 2006;82(1):102-7.

6. Meier-Kriesche HU, Ojo AO, Leichtman AB, Punch JD, Hanson JA, Cibrik DM, et al. Effect of mycophenolate mofetil on long-term outcomes in African Americans renal transplant patients. J Am Soc Nephrol. 2000;11(12):2366-70. 
7. Srinivas TR, Kaplan B, Schold JD, Meier-Kriesche HU The impact of mycophenolate mofetil on long-term outcomes in kidney transplantation. Transplantation. 2005;80 (2 Suppl): S211-220

8. Miller J, Mendez R, Pirsch JD, Jensik SC. Safety and efficacy of tacrolimus in combination with mycophenolate mofetil (MMF) in cadaveric renal transplant recipients. FK506/MMF Dose-Ranging Kidney Transplant Study Group. Transplantation. 2000; 69(5):875-80.

9. Squifflet JP, Bäckman L, Claesson K, Dietl KH, Ekberg H, Forsythe JL, et al; European Tacrolimus-MMF Renal Study Group. Dose optimization of mycophenolate mofetil when administered with a low dose of tacrolimus in cadaveric renal transplant recipients. : Transplantation. 2001;72(1):63-9.

10. Knoll GA, MacDonald I, Khan A, Van Walraven C. Mycophenolate mofetil dose reduction and the risk of acute rejection after renal transplantation. J Am Soc Nephrol. 2003;14(9):2381-6.

11. Davies NM, Grinyó J, Heading R, Maes B, Meier-Kriesche HU, Oellerich M. Gastrointestinal side effects of mycophenolic acid in renal transplant patients: a reappraisal. Nephrol Dial Transplant. 2007;22(9):2440-8.

12. Bunnapradist S, Neri L, Wong W, Lentine KL, Burroughs TE, Pinsky BW, et al. Incidence and risk factors for diarrhea following kidney transplantation and association with graft loss and mortality. Am J Kidney Dis. 2008;51(3):478-86.

13. Vinik AI, Maser RE, Mitchell BD, Freeman R. Diabetic autonomic neuropathy. Diabetes Care. 2003;26(5):1553-79.

14. Khurana A, McCuskey CF, Slavcheva EG. Orthostatic hypotension in kidney pancreas transplant patients and its relation to preexisting autonomic neuropathy. Exp Clin Transplant. 2008;6(2)127-31.

15. Salvadori M, Holzer H, de Mattos A, Sollinger H, Arns W, Oppenheimer F, et al;. The ERL B301 Study Groups. Enteric-coated mycophenolate sodium is therapeutically equivalent to mycophenolate mofetil in de novo renal transplant patients. Am J Transplant. 2004;4(2):231-6.
16. Budde K, Curtis J, Knoll G, Chan L, Neumayer HH, Seifu Y, et al; ERL B302 Study Group. Enteric-coated mycophenolate sodium can be safely administered in maintenance renal transplant patients: results of a 1-year study. Am J Transplant. 2004;4(2):237-43.

17. Arns W, Breuer S, Choudhury S, Taccard G, Lee J, Binder V, et al. Enteric-coated mycophenolate sodium delivers bioequivalent MPA exposure compared with mycophenolate mofetil. Clin Transplant. 2005;19(2):199-206.

18. Tedesco-Silva H, Bastien MC, Choi L, Felipe C, Campestrini J, Picard F, et al. Mycophenolic acid metabolite profile in renal transplant patients receiving enteric-coated mycophenolate sodium or myciphenolate mofetil. Transplant Proc. 2005;37(2):852-5.

19. Darji P, Vijayaraghavan R, Thiagarajan CM, Sharma RK, Subbarao B, Pishardy $\mathrm{R}$, et al. Conversion from mycophenolate mofetil to enteric-coated mycophenolate sodium in renal transplant recipients with gastrointestinal tract disorders. Transplant Proc. 2008;40(7):2262-7.

20. Chan L, Mulgaonkar S, Walker R, Arns W, Ambuhl P, Schiavelli R. Patient-reported gastroinstestinal symptom burden and health-related quality of life following conversion from Mycophenolate Mofetil to enteric-coated Mycophenolate Sodium. Transplantation. 2006;81(9):1290-7.

21. Budde K, Glander P, Krämer BK, Fischer W, Hoffmann U, Bauer S, et al. Conversion from mycophenolate mofetil to enteric-coated mycophenolate sodium in maintenance renal transplant recipients receiving tacrolimus: clinical, pharmacokinetic, and pharmacodynamic outcomes. Transplantation. 2007;83(4):417-24.

22. Machnicki G, Ricci JF, Brennan DC, Schnitzler MA. Economic impact and longterm graft outcomes of mycophenolate mofetil dosage modifications following gastrointestinal complications in renal transplant recipients. Pharmacoeconomics. 2008;26(11):951-67.

23. Lo A, Stratta RJ, Egidi MF, Shokouh-Amiri MH, Grewal HP, Kisilisik AT, et al. Patterns of cytomegalovirus infection in simultaneous kidney-pancreas transplant recipients receiving tacrolimus, mycophenolate mofetil, and prednisone with ganciclovir prophylaxis. Transpl Infect Dis. 2001;3(1):8-15. 